\title{
Surface term, Virasoro algebra, and Wald entropy of black holes in higher-curvature gravity
}

\author{
Shao-Jun Zhang* and Bin Wang ${ }^{+}$ \\ Department of Physics, INPAC, Shanghai Jiao Tong University, Shanghai 200240, China
}

(Dated: October 30, 2018)

\begin{abstract}
Recently, in the Einstein gravity, Majhi and Padmanabhan proposed a straightforward and transparent way of obtaining the Bekenstein-Hawking entropy by using an approach based on the Virasoro algebra and central charge. In this work, we generalize their approach to the modified gravity with higher-curvature corrections and show that their approach can successfully lead to the corresponding Wald entropy in the higher-curvature gravity. Our result shows that the approach is physically general.
\end{abstract}

PACS numbers: 04.62.+v, 04.60.-m

\section{INTRODUCTION}

It is well known that black holes act as a bridge connecting general relativity and quantum mechanics. Studying various properties of black holes can provide us with a possible way to understand the underlying quantum gravity. One of the most remarkable features of a black hole is its thermodynamical ensemble [1 4], which has the concept of not only temperature, but also entropy. In classical general relativity, it is usually hard to have a statistical understanding of black hole entropy. In the past decades, various attempts have been made to interpret the microscopic origin of black hole entropy. One of them, is to use Virasoro algebra and the Cardy formula [5 - 7] to explain the statistical meaning of the entropy [8, [9]. This method was based on the original approach by Brown and Henneaux [10] in studying the asymptotical symmetry group of $(2+1)$ dimensional gravity in asymptotically anti-de Sitter spacetime. The basic idea of this method is to define the corresponding Noether charges and Lie bracket among some chosen diffeomorphisms; then the Lie bracket turns out to be the well-known Virasoro algebra with central extension. One can read off the deduced central charge and the zero-mode eigenvalues of the Fourier modes of the charge. Substituting them into the Cardy formula, Bekenstein-Hawking entropy can be obtained.

*Electronic address: sjzhang84@sjtu.edu.cn

${ }^{\dagger}$ Electronic address: wang_b@sjtu.edu.cn 
Recent development and related references can be found in Ref. [11] and references therein. In this approach, the Noether current was constructed starting from the bulk action, the Einstein-Hilbert action, or the Lovelock action.

Recently, a new approach was proposed by Majhi and Padmanabhan [12] [13]. They introduced the Virasoro algebra defined by the Noether currents corresponding to the surface term of gravitational action, the Gibbons-Hawking term [14], instead of the bulk action. In their work, the diffeomorphisms related to the Noether currents are chosen to be those which leave the nearhorizon form of the metric invariant in some nonsingular coordinates. This new approach is clear and straightforward for obtaining the correct entropy, and does not require any ad hoc prescriptions, such as shifting the zero-mode energy [9] or choosing a parameter in the Fourier modes of $T$ as the surface gravity [15], etc. In the Einstein gravity, this approach leads to the Bekenstein-Hawking entropy without any ambiguities.

The Bekenstein-Hawking entropy, in proportion to the area of the black hole horizon, holds well in Einstein gravity; however, it does not hold in general gravitational theory. For example, in the gravity theory with higher-curvature corrections, the entropy is described by Wald entropy, which contains properly the contributions of higher-curvature corrections [16 19]. It is natural to ask whether the method proposed by Majhi and Padmanabhan can be generalized to produce the Wald entropy in the gravity theory with higher-curvature corrections. This will serve as the main motivation of the present paper. We will study the higher-curvature gravitational theories, including Gauss-Bonnet and third-order Lovelock gravity. We will show that the Virasoro algebra, with the information of higher-curvature terms defined by the Noether currents corresponding to the surface term of gravitational action has a central extension that indeed leads to the Wald entropy. We will argue that the method proposed by Majhi and Padmanabhan is general, which can provide deeper insight into the nature of the entropy.

The organization of the paper is as follows: In the next section, we will give a brief review on general formulas of Noether current constructed from the surface term of gravitational action. In Sec. III, we will first give general derivations of related vector fields for static spherical spacetimes, and then apply three different gravitational theories, namely the Einstein, Gauss-Bonnet, and third-order Lovelock theories. We will derive the charge and Virasoro algebra starting from the corresponding surface term of the gravitational action and then use the Cardy formula to obtain the entropy in different gravitational theories. We will summarize our results in the last section. 


\section{GENERAL FORMULAS OF NOETHER CURRENT FROM THE SURFACE TERM}

In this section, let us briefly review the general formulas of Noether current constructed from the surface term of gravitational action [11 13]. Let us consider a general surface term as

$$
\begin{aligned}
I_{B} & =\frac{1}{8 \pi G} \int_{\partial \mathcal{M}} d^{n-1} x \sqrt{\gamma} \mathcal{L}_{B} \\
& =\frac{1}{8 \pi G} \int_{\mathcal{M}} d^{n} x \sqrt{g} \nabla_{a}\left(\mathcal{L}_{B} N^{a}\right),
\end{aligned}
$$

where $N^{a}$ is the unit normal vector of the boundary $\partial \mathcal{M}, g_{a b}$ denotes the bulk metric and $\gamma_{a b}$ denotes the induced boundary metric. The Noether current we will study corresponds to dif-

feomorphism transformation. For convenience, we define $A^{a} \equiv \frac{1}{8 \pi G} \mathcal{L}_{B} N^{a}$, so the $n$-dimensional Lagrangian density is $\sqrt{g} \mathcal{L}=\sqrt{g} \nabla_{a} A^{a}$. Under a general diffeomorphism $x^{a} \rightarrow x^{a}+\xi^{a}$, the Lagrangian density $\sqrt{g} \mathcal{L}$ changes by

$$
\delta_{\xi}(\sqrt{g} \mathcal{L}) \equiv £_{\xi}(\sqrt{g} \mathcal{L})=\sqrt{g} \nabla_{a}\left(\mathcal{L} \xi^{a}\right)
$$

In deriving the above equation, we have used the formulas $£_{\xi}(\sqrt{g})=\sqrt{g} \nabla_{a} \xi^{a}$ and $£_{\xi}(\mathcal{L})=\xi^{a} \nabla_{a} \mathcal{L}$. On the other hand, we also have

$$
\begin{aligned}
\delta_{\xi}\left(\sqrt{g} \nabla_{a} A^{a}\right) & =£_{\xi}\left[\partial_{a}\left(\sqrt{g} A^{a}\right)\right] \\
& =\sqrt{g} \nabla_{a}\left[\nabla_{b}\left(A^{a} \xi^{b}\right)-A^{b} \nabla_{b} \xi^{a}\right],
\end{aligned}
$$

where we have used the formula $\nabla_{a} A^{a}=\frac{1}{\sqrt{g}} \partial_{a}\left(\sqrt{g} A^{a}\right)$.

Equating Eqs. (2) and (3), we get the conserved Noether current

$$
J^{a}[\xi]=\mathcal{L} \xi^{a}-\nabla_{b}\left(A^{a} \xi^{b}\right)+A^{b} \nabla_{b} \xi^{a}
$$

Substituting $\mathcal{L}=\nabla_{a} A^{a}$ into the above equation, the Noether current can be rewritten as

$$
J^{a}[\xi]=\nabla_{b} J^{a b}[\xi]=\nabla_{b}\left[\xi^{a} A^{b}-\xi^{b} A^{a}\right]
$$

Usually, $J^{a b}$ is called the Noether potential. With the explicit expression $A^{a}=\frac{1}{8 \pi G} \mathcal{L}_{\mathcal{B}} N^{a}$, we get the final form of the Noether current constructed from the surface term

$$
J^{a}[\xi]=\nabla_{b} J^{a b}[\xi]=\frac{1}{8 \pi G} \nabla_{b}\left[\mathcal{L}_{B}\left(\xi^{a} N^{b}-\xi^{b} N^{a}\right)\right] .
$$

The corresponding charge is defined as

$$
Q[\xi]=\frac{1}{2} \int_{\Sigma} \sqrt{h} d \Sigma_{a b} J^{a b}
$$


where $d \Sigma_{a b}=-d^{n-1} x\left(N_{a} M_{b}-N_{b} M_{a}\right)$ is the surface element of the $(n-2)$-dimensional surface $\Sigma$, and $h$ is the determinant of the corresponding induced metric. $N^{a}$ and $M^{a}$ are chosen to be spacelike and timelike, respectively. In our discussion, $\Sigma$ will be near the horizon $\mathcal{H}$.

Following Ref. [11], we define a Lie bracket for the charges

$$
\begin{aligned}
{\left[Q_{1}, Q_{2}\right]: } & =\left(\delta_{\xi_{1}} Q\left[\xi_{2}\right]-\delta_{\xi_{2}} Q\left[\xi_{1}\right]\right) \\
& =\int_{\Sigma} \sqrt{h} d \Sigma_{a b}\left[\xi_{2}^{a} J^{b}\left[\xi_{1}\right]-\xi_{1}^{a} J^{b}\left[\xi_{2}\right]\right]
\end{aligned}
$$

which will lead to the usual Virasoro algebra with central extension. As we will see soon, with the deduced central charge and the Cardy formula, the entropy of black holes can be derived.

\section{ENTROPY OF BLACK HOLES FROM THE CARDY FORMULA}

To derive the Noether charge and Virasoro algebra, we first need to identify appropriate diffeomorphisms, the related vector fields $\xi^{a}$. In Ref. [12], the authors did this in the explicit Rindler metric. Here, we give a more abstract derivation of the vector fields $\xi^{a}$.

To be transparent, we only consider static spherical black holes with the general form of the metric

$$
d s^{2}=-f(r) d t^{2}+\frac{d r^{2}}{f(r)}+r^{2} \Omega_{i j}(x) d x^{i} d x^{j},
$$

where $\Omega_{i j}(x)$ is the $(n-2)$-dimensional space and $h_{i j}=r^{2} \Omega_{i j}$. The horizon is located at $r=r_{h}$, satisfying $f\left(r_{h}\right)=0$. The two normal vector fields $N^{a}, M^{a}$ are

$$
N^{a}=\left(0, \sqrt{-f\left(\rho+r_{h}\right)}, 0, \cdots, 0\right), \quad M^{a}=\left(\sqrt{\frac{1}{f\left(\rho+r_{h}\right)}}, 0, \cdots, 0\right) .
$$

To study the near-horizon structure, it is convenient to define $r \equiv \rho+r_{h}$, and in the near-horizon limit, $\rho \rightarrow 0$. The metric then becomes

$$
d s^{2}=-f\left(\rho+r_{h}\right) d t^{2}+\frac{d \rho^{2}}{f\left(\rho+r_{h}\right)}+\left(\rho+r_{h}\right)^{2} \Omega_{i j}(x) d x^{i} d x^{j} .
$$

The function $f\left(\rho+r_{h}\right)$ in the near-horizon limit can be expanded as $f\left(\rho+r_{h}\right)=2 \kappa f^{\prime}\left(r_{h}\right) \rho+$ $\frac{1}{2} f^{\prime \prime}\left(r_{h}\right) \rho^{2}+\cdots$ with surface gravity $\kappa=\frac{f^{\prime}\left(r_{h}\right)}{2}$. Then it is easy to see that the $t-\rho$ part of the above metric becomes Rindler in the near-horizon limit, $d s_{t=\rho}^{2}=-2 \kappa \rho d t^{2}+\frac{1}{2 \kappa \rho} d \rho^{2}$, if we only keep the first-order term of the expansion of $f\left(\rho+r_{h}\right)$. In Ref. [12], the authors started from a Rindler metric. Here, we keep the full metric in the process of derivation and only do the near-horizon limit in the end. Introducing Bondi-like coordinates by the transformation [12]

$$
d u=d t-\frac{d \rho}{f\left(\rho+r_{h}\right)},
$$


the metric is transformed to

$$
d s^{2}=-f\left(\rho+r_{h}\right) d u^{2}-2 d u d \rho+\left(\rho+r_{h}\right)^{2} \Omega_{i j}(x) d x^{i} d x^{j} .
$$

To obtain the Noether current, we choose the vector fields $\xi^{a}$ which can leave the horizon structure invariant

$$
\begin{aligned}
& 0=£_{\xi} g_{\rho \rho}=-2 \partial_{\rho} \xi^{\mu} \\
& 0=£_{\xi} g_{u \rho}=-f\left(\rho+r_{h}\right) \partial_{\rho} \xi^{u}-\partial_{\rho} \xi^{\rho}-\partial_{u} \xi^{u}
\end{aligned}
$$

Solving the above equations, we obtain

$$
\xi^{u}=F(u, x), \quad \xi^{\rho}=-\rho \partial_{u} F(u, x),
$$

while the other components vanish. It is easy to check that $£_{\xi} g_{u u}=\mathcal{O}(\rho)$, so that the condition $£_{\xi} g_{u u}=0$ is satisfied near the horizon. This shows that the derived vector fields are indeed what we want. Returning to the original coordinates $(t, \rho)$, these vector fields become

$$
\xi^{t}=T-\frac{1}{f\left(r_{h}+\rho\right)} \partial_{t} T, \quad \xi^{\rho}=-\rho \partial_{t} T, \quad T(t, \rho, x) \equiv F(u, x) .
$$

For a given $T$, from Eqs. (16) and (7), we have expressions of the corresponding vector field $\xi^{a}$ and the charge $Q$, respectively.

Expanding $T$ in terms of the basis functions $T_{m}$

$$
T=\sum_{m} A_{m} T_{m}, \quad A_{m}^{*}=A_{-m}
$$

and substituting it into Eqs. (16) and (7), we get corresponding expansions for $\xi^{a}$ and $Q$ in the forms of Eqs. (16) and (7), where $T$ is replaced by $T_{m} . T_{m}$ is chosen to be the basis that yields the resulting $\xi_{m}^{a}$, obeying the algebra isomorphic to Diff $S^{1}$ :

$$
i\left\{\xi_{m}, \xi_{n}\right\}^{a}=(m-n) \xi_{m+n}^{a},
$$

with $\{$,$\} being the Lie bracket. A standard choice is [15]$

$$
T_{m}=\frac{1}{\alpha} \exp [i m(\alpha t+g(\rho)+p \cdot x)]
$$

where $p$ is an integer and $g(\rho)$ as a function that is regular at the horizon. The value $\alpha$ is a parameter which needs to be chosen according to the surface gravity in Refs. [11, 15], but it is arbitrary in the present approach and will not affect the final results. 


\section{A. Warmup: Four-dimensional Schwarzschild black hole}

As a warmup, let us first apply the above formulas to a four-dimensional Schwarzschild black hole in Einstein gravity. In the local Rindler frame, the results were shown in Refs. [12] [13].

The surface term of the gravitational action is the well-known Gibbons-Hawking term [14]

$$
\mathcal{L}_{B}=K
$$

with $K=-\nabla_{a} N^{a}$ being the trace of the extrinsic curvature of the boundary $\partial \mathcal{M}$. Considering $f(r)=1-\frac{2 M}{r}$ and $\Omega_{i j} d x^{i} d x^{j}=d \Omega_{2}^{2}$ for the Schwarzschild black hole, we have the horizon $\mathcal{H}$ at $r_{h}=2 M$, and the new form of the metric becomes

$$
d s^{2}=-\frac{\rho}{2 M+\rho} d t^{2}+\frac{2 M+\rho}{\rho} d \rho^{2}+(2 M+\rho)^{2} d \Omega_{2}^{2}
$$

where $\rho=0$ represents the horizon. In Bondi-like coordinates defined by the transformation [Eq. (12)], we have

$$
d u=d t-\frac{2 M+\rho}{\rho} d \rho
$$

and the metric becomes

$$
d s^{2}=-\frac{\rho}{2 M+\rho} d u^{2}-2 d u d \rho+(2 M+\rho)^{2} d \Omega_{2}^{2}
$$

In the coordinates $(t, \rho), \xi^{a}$ are chosen to be

$$
\xi^{t}=T-(2 M+\rho) \partial_{t} T, \quad \xi^{\rho}=-\rho \partial_{t} T
$$

where $T(t, \rho, x) \equiv F(u, x)$.

Now we are ready to derive the Noether current, and then the Virasoro algebra. For the Schwarzschild black hole metric, we have

$$
\begin{aligned}
N^{a} & =\left(0, \sqrt{\frac{\rho}{\rho+2 M}}, 0,0\right), \quad M^{a}=\left(\sqrt{\frac{\rho+2 M}{\rho}}, 0,0,0\right), \\
K & =-\frac{M+2 \rho}{\sqrt{\rho}(2 M+\rho)^{3 / 2}} .
\end{aligned}
$$

The charge at the near-horizon limit $\rho \rightarrow 0$ is

$$
Q[\xi]=\frac{1}{8 \pi G} \int_{\mathcal{H}} \sqrt{h} d^{2} x\left[\kappa T-\frac{1}{2} \partial_{t} T\right],
$$


where $\kappa=\frac{1}{4 M}=\frac{f^{\prime}\left(r_{h}\right)}{2}$ is the surface gravity of the black hole. For $T=T_{m}, T_{n}$, the commutator [Eq. (8)] can be directly calculated, and the result in the near-horizon limit $\rho \rightarrow 0$ is

$$
\begin{aligned}
{\left[Q_{m}, Q_{n}\right]=\frac{1}{8 \pi G M} \int_{\mathcal{H}} \sqrt{h} d^{2} x\left[\kappa\left(T_{m} \partial_{t} T_{n}-T_{n} \partial_{t} T_{m}\right) .\right.} \\
-\frac{1}{2}\left(T_{m} \partial_{t}^{2} T_{n}-T_{n} \partial_{t}^{2} T_{m}\right) \\
\left.+\frac{1}{4 \kappa}\left(\partial_{t} T_{m} \partial_{t}^{2} T_{n}-\partial_{t} T_{n} \partial_{t}^{2} T_{m}\right)\right] .
\end{aligned}
$$

In doing these calculations, we actually do not need the explicit form of $f(r)$. From Eqs. (26) and (27), we can see that $f\left(\rho+r_{h}\right)$ only affects the surface gravity $\kappa$; the second- and higher-order terms in the expansion of $f\left(\rho+r_{h}\right)$ give no contributions to the final results. So if we start from the Rindler approximation of the full metric, we can still get the same result. We will show that this is also true for the Gauss-Bonnet and third-order Lovelock cases discussed in the next two subsections. The extension to cases with a cosmological constant and charges is straightforward. The charge and Virasoro algebra will take the same forms as Eqs. (26) and (27), only with a different surface gravity.

By substituting Eq. (19) into Eqs. (26) and (27) and finishing the integration over a cross-section area $A$, we arrive at the final explicit expressions:

$$
\begin{aligned}
& Q_{m}=\frac{A}{8 \pi G} \frac{\kappa}{\alpha} \delta_{m, 0}, \\
& {\left[Q_{m}, Q_{n}\right]=\frac{i \kappa A}{8 \pi G \alpha}(m-n) \delta_{m+n, 0}-i m^{3} \frac{\alpha A}{16 \pi G \kappa} \delta_{m+n, 0} .}
\end{aligned}
$$

Then we can obtain the central term in the algebra,

$$
\begin{aligned}
K\left[\xi_{m}, \xi_{n}\right] & =\left[Q_{m}, Q_{n}\right]+i(m-n) Q\left[\xi_{m+n}\right] \\
& =-i m^{3} \frac{A}{16 \pi G} \frac{\alpha}{\kappa} \delta_{m+n, 0} .
\end{aligned}
$$

From the central term, we can read off the central charge $C$ and the zero mode $Q_{0}$ as

$$
\frac{C}{12}=\frac{A}{16 \pi G} \frac{\alpha}{\kappa}, \quad Q_{0} \equiv Q\left[\xi_{0}\right]=\frac{A}{8 \pi G} \frac{\kappa}{\alpha} .
$$

Using the Cardy formula [5 7], we finally obtain the entropy

$$
S=2 \pi \sqrt{\frac{C Q_{0}}{6}}=\frac{A}{4 G}
$$

which is exactly the Bekenstein-Hawking entropy, as expected. If we include a cosmological constant or charge, the entropy formula will remain the same. 


\section{B. Entropy of a Gauss-Bonnet black hole}

Now we extend the approach to derive the entropy of a Gauss-Bonnet black hole. The surface term of the gravitation action now takes the form [20] [21]

$$
\mathcal{L}_{B}=K+2 \alpha_{2}\left(J-2 \hat{G}_{a b}^{(1)} K^{a b}\right)
$$

where $\alpha_{2}$ is the coefficient of the Gauss-Bonnet term, $\hat{G}_{a b}^{(1)}$ denotes the $(n-1)$-dimensional Einstein tensor of the induced metric $\gamma_{a b}$, and $J$ is the trace of the following tensor

$$
J_{a b}=\frac{1}{3}\left(2 K K_{a c} K_{b}^{c}+K_{c d} K^{c d} K_{a b}-2 K_{a c} K^{c d} K_{d b}-K^{2} K_{a b}\right) .
$$

A family of general static spherical Gauss-Bonnet black hole solutions [22] [23] takes the same form as Eq. (9), but with

$$
f(r)=k+\frac{r^{2}}{4 \alpha_{2}}\left(1-\sqrt{1+\frac{4}{3} \alpha_{2} \Lambda+8 \alpha_{2} \frac{\mu}{r^{4}}}\right) .
$$

The term $\Omega_{i j}$ denotes the metric of an $(n-2)$-dimensional hypersurface with the constant curvature scalar $(n-2)(n-3) k,(k=0, \pm 1)$, and $\mu$ and $\Lambda$ relate to the gravitational mass and the cosmological constant, respectively. Without loss of generality, we will focus on the case with $n=5$.

Now we proceed to calculate the charge and Virasoro algebra for Gauss-Bonnet black holes with different topologies. In the near-horizon limit, the charge and Virasoro algebra can be written in a unified form:

$$
\begin{gathered}
Q[\xi]=\frac{1}{8 \pi G}\left(1+\frac{12 k \alpha_{2}}{r_{h}^{2}}\right) \int_{\mathcal{H}} \sqrt{h} d^{2} x\left[\kappa T-\frac{1}{2} \partial_{t} T\right] \\
{\left[Q_{m}, Q_{n}\right]=\frac{1}{8 \pi G}\left(1+\frac{12 k \alpha_{2}}{r_{h}^{2}}\right) \int_{\mathcal{H}} \sqrt{h} d^{2} x\left[\kappa\left(T_{m} \partial_{t} T_{n}-T_{n} \partial_{t} T_{m}\right) .\right.} \\
-\frac{1}{2}\left(T_{m} \partial_{t}^{2} T_{n}-T_{n} \partial_{t}^{2} T_{m}\right) \\
\left.+\frac{1}{4 \kappa}\left(\partial_{t} T_{m} \partial_{t}^{2} T_{n}-\partial_{t} T_{n} \partial_{t}^{2} T_{m}\right)\right],
\end{gathered}
$$

where the surface gravity $\kappa=\frac{f^{\prime}\left(r_{h}\right)}{2}$. During our calculations, we keep all expansion terms in the metric function and take the near-horizon limit at the end. Comparing this with the Einstein case [Eq. (27)], we see that an additional factor $\left(1+\frac{12 k \alpha_{2}}{r_{h}^{2}}\right)$ appears due to the contribution of the Gauss-Bonnet term.

Choosing $T_{m}$ as Eq. (19) and substituting it into the above equations, we get

$$
\begin{aligned}
& Q_{m}=\frac{A}{8 \pi G}\left(1+\frac{12 \alpha_{2} k}{r_{h}^{2}}\right) \frac{\kappa}{\alpha} \delta_{m, 0} \\
& {\left[Q_{m}, Q_{n}\right]=-\frac{i \kappa A}{8 \pi G \alpha}\left(1+\frac{12 \alpha_{2} k}{r_{h}^{2}}\right)(m-n) \delta_{m+n, 0}-i m^{3} \frac{\alpha A}{16 \pi G \kappa}\left(1+\frac{12 \alpha_{2} k}{r_{h}^{2}}\right) \delta_{m+n, 0} .}
\end{aligned}
$$


The central term reads

$$
\begin{aligned}
K\left[\xi_{m}, \xi_{n}\right] & =\left[Q_{m}, Q_{n}\right]+i(m-n) Q\left[\xi_{m+n}\right] \\
& =-i m^{3} \frac{A}{16 \pi G}\left(1+\frac{12 \alpha_{2} k}{r_{h}^{2}}\right) \frac{\alpha}{\kappa} \delta_{m+n, 0},
\end{aligned}
$$

from which we can read off the central charge $C$ and zero mode $Q_{0}$ as

$$
\frac{C}{12}=\frac{A}{16 \pi G}\left(1+\frac{12 \alpha_{2} k}{r_{h}^{2}}\right) \frac{\alpha}{\kappa}, \quad Q_{0} \equiv Q\left[\xi_{0}\right]=\frac{A}{8 \pi G}\left(1+\frac{12 \alpha_{2} k}{r_{h}^{2}}\right) \frac{\kappa}{\alpha} .
$$

Using the Cardy formula, we obtain the entropy

$$
S=2 \pi \sqrt{\frac{C Q_{0}}{6}}=\frac{A}{4 G}\left(1+\frac{12 \alpha_{2} k}{r_{h}^{2}}\right),
$$

which is exactly the Wald entropy of the Gauss-Bonnet black holes [16 19, 24]. For the flat case with $k=0$, the entropy in Eq. (40) reduces to the Bekenstein-Hawking entropy as expected, because at this time $\Omega_{i j} d x^{i} d x^{j}$ is flat and will not cause corrections to the Bekenstein-Hawking entropy. This result coincides with the discovery in Ref. [24] from the Wald entropy formula or thermodynamics approach.

\section{Entropy of a third-order Lovelock black hole}

Let us go further to see if this approach can produce the correct entropy for a more general gravitational theory, the third-order Lovelock gravity. The boundary term now reads as [25]

$$
\begin{aligned}
\mathcal{L}_{B}= & K+2 \alpha_{2}\left(J-2 \hat{G}_{a b}^{(1)} K^{a b}\right)+3 \alpha_{3}\left(P-2 \hat{G}_{a b}^{(2)} K^{a b}-12 \hat{R}_{a b} J^{a b}\right. \\
& \left.+2 \hat{R} J-4 K \hat{R}_{a b c d} K^{a c} K^{b d}-8 \hat{R}_{a b c d} K^{a c} K^{b} K^{e d}\right) .
\end{aligned}
$$

Here, $\hat{R}_{a b c d}$ is the intrinsic Riemannian tensor constructed from the boundary metric $\gamma_{a b}$. $\hat{G}_{a b}^{(2)}$ is the second-order Lovelock tensor of $\gamma_{a b}$; that is,

$$
\hat{G}_{a b}^{(2)}=2\left(\hat{R}_{a c d e} \hat{R}_{b}^{c d e}-2 \hat{R}_{a c b d} \hat{R}^{c d}-2 \hat{R}_{a c} \hat{R}_{b}^{c}+\hat{R} \hat{R}_{a b}\right)-\frac{1}{2} \mathcal{L}_{2} \gamma_{a b}
$$

with $\mathcal{L}_{2}$ being the Gauss-Bonnet term $\mathcal{L}_{2}=\hat{R}_{a b c d} \hat{R}^{a b c d}-4 \hat{R}_{a b} \hat{R}^{a b}+\hat{R}^{2} . P$ is the trace of the following tensor:

$$
\begin{aligned}
P_{a b}=\frac{1}{5}\{[ & \left.K^{4}-6 K^{2} K^{c d} K_{c d}+8 K K_{c d} K_{e}^{d} K^{e c}-6 K_{c d} K^{d e} K_{e f} K^{f c}+3\left(K_{c d} K^{c d}\right)^{2}\right] K_{a b} \\
& -\left(4 K^{3}-12 K K_{e d} K^{e d}+8 K_{d e} K_{f}^{e} K^{f d}\right) K_{a c} K_{b}^{c}-24 K K_{a c} K^{c d} K_{d e} K_{b}^{e} \\
& \left.+\left(12 K^{2}-12 K_{e f} K^{e f}\right) K_{a c} K^{c d} K_{d b}+24 K_{a c} K^{c d} K_{d e} K^{e f} K_{b f}\right\}
\end{aligned}
$$


Without loss of generality, we will concentrate on the seven-dimensional static, spherical black hole solutions of the third-order Lovelock gravity. General black hole solutions in third-order Lovelock gravity with arbitrary coefficients $\alpha_{2}$ and $\alpha_{3}$ are rather complicated to obtain. In Refs. [26, 27], it was found that an explicit solution of a black hole exists when the two coefficients satisfy $2 \alpha_{2}^{2}=\alpha_{3}=\frac{\tilde{\alpha}^{2}}{72}$ with the metric function in Eq. (9) in the form

$$
f(r)=k+\frac{r^{2}}{\tilde{\alpha}}\left[1-\left(1+\frac{\Lambda \tilde{\alpha}}{5}+\frac{3 \tilde{\alpha} \mu}{5 r^{6}}\right)^{1 / 3}\right] .
$$

The term $\Omega_{i j} d x^{i} d x^{j}$ denotes an $(n-2)$-dimensional hypersurface with the constant curvature scalar $(n-2)(n-3) k,(k=0, \pm 1)$. Following the approach we discussed above, we can obtain unified expressions of the charge and the Virasoro algebra for different topological spacetimes:

$$
\begin{aligned}
Q[\xi]=\frac{1}{8 \pi G}\left(1+\frac{40 \alpha_{2} k}{r_{h}^{2}}+\frac{360 \alpha_{3} k^{2}}{r_{h}^{4}}\right) \int_{\mathcal{H}} \sqrt{h} d^{2} x\left[\kappa T-\frac{1}{2} \partial_{t} T\right] \\
\begin{aligned}
{\left[Q_{m}, Q_{n}\right]=\frac{1}{8 \pi G}\left(1+\frac{40 \alpha_{2} k}{r_{h}^{2}}+\frac{360 \alpha_{3} k^{2}}{r_{h}^{4}}\right) } & \int_{\mathcal{H}} \sqrt{h} d^{2} x\left[\kappa\left(T_{m} \partial_{t} T_{n}-T_{n} \partial_{t} T_{m}\right) .\right. \\
& -\frac{1}{2}\left(T_{m} \partial_{t}^{2} T_{n}-T_{n} \partial_{t}^{2} T_{m}\right) \\
& \left.+\frac{1}{4 \kappa}\left(\partial_{t} T_{m} \partial_{t}^{2} T_{n}-\partial_{t} T_{n} \partial_{t}^{2} T_{m}\right)\right],
\end{aligned}
\end{aligned}
$$

where the surface gravity $\kappa=\frac{f^{\prime}\left(r_{h}\right)}{2}$, and the additional factor changes to be $\left(1+\frac{40 \alpha_{2} k}{r_{h}^{2}}+\frac{360 \alpha_{3} k^{2}}{r_{h}^{4}}\right)$, containing the contribution of the third Lovelock term. After using the Cardy formula, we obtain the entropy of the third Lovelock black hole,

$$
S=\frac{A}{4 G}\left(1+\frac{40 \alpha_{2} k}{r_{h}^{2}}+\frac{360 \alpha_{3} k^{2}}{r_{h}^{4}}\right),
$$

which is consistent with the result derived in Ref. [26, 27] in the form of the Wald entropy formula. Here we cannot expect the above entropy to reduce to the Gauss-Bonnet case [Eq. (40)] by taking the limit $\alpha_{3}=0$, because in our considered case, $\alpha_{2}$ and $\alpha_{3}$ are not independent.

Although we only consider a special case with two coefficients satisfying a particular relation, we want to indicate that in calculating the Noether charge [Eq. (45)] and Virasoro algebra [Eq. 460)], we actually do not need the explicit form of the function $f(r)$ in Eq. (44)-what we need is only the form of the metric Eq. (9). So, for more general solutions of the black hole with the metric in the form of Eq. (9), we can get a similar result for different surface gravity and different correction terms in the entropy expression. 


\section{SUMMARY}

In this paper, we have generalized the method proposed in Refs. [12, 13] to derive the entropy of black holes in Gauss-Bonnet and third-order Lovelock gravity. We have shown that in addition to reproducing the correct Bekenstein-Hawking entropy in the Einstein gravity, the method can produce the Wald entropy in the general theories of gravity with higher-curvature corrections. This shows that the approach based on the Virasoro algebra and central charge from the surface term of gravitational action is general, which can provide deeper insight into the nature of the entropy.

In our calculation we noticed that the expressions of Noether charge and Virasoro algebra only depend on the form of the metric [Eq. (9)], and do not heavily depend on the explicit expression in the metric function. This tells us that the method to derive the entropy can even work for more complicated expressions in the metric form of Eq. (9). It is interesting to ask whether the method is still effective for rotating black holes. More investigation in this direction is called for, as it can show us the universality of the method.

[1] J.D. Bekenstein, Black holes and entropy, Phys. Rev. D 7 (1973) 2333.

[2] J.M. Bardeen, B. Carter and S.W. Hawking, The four laws of black hole mechanics, Commun. Math. Phys. 31 (1973) 161.

[3] S.W. Hawking, Black hole explosions, Nature 248 (1974) 30.

[4] S.W. Hawking, Particle creation by black holes, Commun. Math. Phys. 43 (1975) 199; Erratum-ibid. 46 (1976) 206.

[5] J.L. Cardy, Operator content of two-dimensional conformally invariant theories, Nucl. Phys. B 270 (1986) 186.

[6] H.W. Bloete, J.L. Cardy and M.P. Nightingale, Conformal invariance, the central charge, and universal finite size amplitudes at criticality, Phys. Rev. Lett. 56 (1986) 742.

[7] S. Carlip, What we don't know about BTZ black hole entropy, Class. Quant. Grav. 15 (1998) 3609 arXiv:hep-th/9806026.

[8] S. Carlip, Black hole entropy from conformal field theory in any dimension, Phys. Rev. Lett. 82 (1999) 2828 arXiv:hep-th/9812013.

[9] S. Carlip, Entropy from conformal field theory at Killing horizons, Class. Quant. Grav. 16 (1999) 3327 arXiv:gr-qc/9906126.

[10] J.D. Brown and M. Henneaux, Central charges in the canonical realiztion of asymptotic symmetries: an example from three-dimensional gravity, Commun. Math. Phys. 104 (1986) 207.

[11] B.R. Majhi and T. Padmanabhan, Noether current, horizon Virasoro algebra and entropy, Phys. Rev. 
D 85 (2012) 084040 arXiv:1111.1809 $[\mathrm{gr}-\mathrm{qc}]]$.

[12] B.R. Majhi and T. Padmanabhan, Noether current from the surface term of gravitational action, Virasoro algebra and horizon entropy, Phys. Rev. D 86 (2012) 101501 (R) [arXiv:1204.1422[gr-qc]].

[13] B.R. Majhi, Noether current of the surface term of Einstein-Hilbert action, Virasoro algebra and entropy, arXiv:1210.6736[gr-qc].

[14] G.W. Gibbons and S.W. Hawking, Action integrals and partition functions in quantum gravity, Phys. Rev. D 15 (1977) 2752.

[15] S. Silva, Black hole entropy and thermodynamics from symmetries, Class. Quant. Grav. 19 (2002) 3947 arXiv:hep-th/0204179.

[16] R.M. Wald, Black hole entropy in the Noether charge, Phys. Rev. D 48 (1993) R3427 arXiv:gr-qc/9307038.

[17] V. Iyer and R.M. Wald, Some properties of Noether charge and a proposal for dynamical black hole entropy, Phys. Rev. D 50 (1994) 846 arXiv:gr-qc/9403028.

[18] V. Iyer and R.M. Wald, A comparison of Noether charge and Euclidean methods for computing the entropy of stationary black holes, Phys. Rev. D 52 (1995) 4430 arXiv:gr-qc/9503052.

[19] T. Jacobson and R.C. Myers, Black hole entropy and higher curvature interactions, Phys. Rev. Lett. 70 (1993) 3684 arXiv:hep-th/9305016.

[20] R.C. Myers, Higher derivative gravity, surface terms and string theory, Phys. Rev. D 36 (1987) 392.

[21] S.C. Davis, Generalized Israel junction conditions for a Gauss-Bonnet brane world, Phys. Rev. D 67 (2003) 024030 arXiv:hep-th/0208205.

[22] D.G. Boulware and S. Deser, String generated gravity models, Phys. Rev. Lett. 55 (1985) 2656.

[23] R.G. Cai, Gauss-Bonnet black holes in AdS spaces, Phys. Rev. D 65 (2002) 084014 arXiv:hep-th/0109133.

[24] T. Clunan, S.F. Ross and D.J. Smith, On Gauss-Bonnet black hole entropy, Class. Quant. Grav. 21 (2004) 3447 arXiv:gr-qc/0402044.

[25] M.H. Dehghani and R.B. Mann, Thermodynamics of Rotating Charged Black Branes in Third Order Lovelock Gravity and the Counterterm Method, Phys. Rev. D 73 (2006) 104003 arXiv:hep-th/0602243

[26] M.H. Dehghani and M. Shamirzaie, Thermodynamics of asymptotically flat charged black holes in third order Lovelock gravity, Phys. Rev. D 72 (2005) 124015 arXiv:hep-th/0506227.

[27] M.H. Dehghani and R. Pourhasan, Thermodynamic Instability of Black Holes of Third Order Lovelock Gravity, Phys. Rev. D 79 (2009) 064015 arXiv:0903.4260[gr-qc]]. 